uninsured, leading to decreased access to care and ability to attend HCV follow-up appointments. ED HCV screening programs are still in their infancy and have opportunities to improve their linkage to care rates. Additional interventions are needed to better connect patients screened positive in the ED to HCV specialist care, preserving equity across racial groups.

3196

Assessing the Relationship between Resilience and Pain Catastrophizing in Patient Reported Outcomes following Total Knee Replacement

Vesta Nwankwo ${ }^{1}$, William Jiranek ${ }^{2}$, Steven George ${ }^{2}$ and Janet Bettger ${ }^{2}$

${ }^{1}$ Duke University and ${ }^{2}$ Duke University Health System

OBJECTIVES/SPECIFIC AIMS: The aim of the present study is to explore the relationships between resilience, pain catastrophizing, and functional outcomes in pre-and post-operative TKR patients. The primary outcome is the ability of the Brief Resilience Scale (BRS) to predict function using the Knee injury and Osteoarthritis Outcome Score Joint Replacement (KOOS, JR.) and overall health on the Patient-Reported Outcome Measurement Information System Global Health (PROMIS GH). METHODS/STUDY POPULATION: Recruited patients will provide informed consent during in-person office visits. At the pre-surgery intake appointment, patients will provide demographic information and complete baseline questionnaires assessing resilience, pain catastrophizing, knee stiffness, pain, and function, and impression of overall health. In-person or electronic follow-up assessments will be administered post-surgery at 6-week, 3month, and 6-month appointments. RESULTS/ANTICIPATED RESULTS: We aim to recruit 150 patients for this study. We do not expect a difference in sociodemographic characteristics of the patient sample. P-values will be based on t-tests and correlations calculated by comparing only non-missing values. Each outcome measure will be examined at each time point and trajectories will be calculated to determine the change in each outcome over time. Using latent class growth modeling we will examine individual change over time for each outcome (BRS, PCS, PROMIS GH, and KOOS JR.), and the association of resilience and pain with change in function (KOOS, JR. total score) and overall health (PROMIS GH). DISCUSSION/SIGNIFICANCE OF IMPACT: To our knowledge, this is the only known study that will compare resilience, pain catastrophizing and longitudinal health outcomes in a single paradigm. Results will hopefully inform the design and implementation of future studies that will assess the impact of preoperative treatment of vulnerable patients. The ultimate goal is to improve functional recovery by using pre-identified psychological constructs as intervention points. Ideally, the same measures would be implemented, however, details of this plan will be established following completion of this study.

3126

Association of ancestral genetic admixture and obesity at 12 months of age

Sahel Hazrati ${ }^{1}$

Inova Health System

OBJECTIVES/SPECIFIC AIMS: We intended to investigate the relationship between children's genetic admixture proportions and the incidence of obesity at 12 months of age METHODS/STUDY POPULATION: 821 twelve month old children were included and their genetic admixture was estimated using the ancestry and kinship toolkit (AKT). Weight for Length Percentile (WFLP) at 12 months of age was categorized as $<95^{\text {th }}$ percentile and $\geq 95^{\text {th }}$ percentile. Multiple logistic regression analysis was performed to calculate odds ratios (ORs) with 95\% confidence intervals (CIs) for association of admixture proportion including EUR, AMR, AFR, EAS and SAS with WFLP categories adjusting for maternal education, birth weight, frequency of breast feeding and juice consumption RESULTS/ANTICIPATED RESULTS: 821 children were included; WFLP $<95^{\text {th }}$ percentile $=671$ $(81.7 \%)$ and $W F L P \geq 95^{\text {th }}$ percentile $=150(18.3 \%)$. Crude odds ratios showed EUR admixture was protective (OR 0.45 (95\% CI 0.27-0.74)) whereas AMR (OR 3.85 (95\% CI 1.92 -7.70)) and AFR (OR 5.70 (95\% CI $2.19-14.85)$ ) were positively associated with obesity. After adjusting for confounding variables, only AFR was associated with WFLP $\geq 95^{\text {th }}($ OR $7.38(95 \%$ CI $2.31-23.59)), A M R$ and EUR were no longer associated with WFLP $\geq 95^{\text {th }}$ DISCUSSION/SIGNIFICANCE OF IMPACT: The AFR admixture proportion remains associated with early childhood obesity after accounting for confounding variables, suggesting the ancestral genetic background may contribute to the differences seen in early childhood obesity

3538

\section{Asthma and Depression in Children: the Role of Family} Structure

Magda Shaheen ${ }^{1}$ and Senait Teklehaimanot

${ }^{1}$ David Geffen School of Medicine at UCLA

OBJECTIVES/SPECIFIC AIMS: Our objective is to examine the relationship between depression, childhood asthma and family structure. METHODS/STUDY POPULATION: We analyzed data from The National Survey of Children's Health 2011-2012 for the variables family structure, parent's smoking, education, poverty, available emotional support, and children's age, gender, race/ethnicity, asthma, physical/mental health, access to care, and insurance. Data were analyzed using multiple logistic regression in STATA 14 (sampling and weight). RESULTS/ANTICIPATED RESULTS: Of the 95,000 participants, $3.8 \%$ had depression, $5.1 \%$ had anxiety, $8.5 \%$ had emotional, developmental, or behavioral problem, $14.6 \%$ had asthma and 19\% were single-parent family. Asthmatics had higher chance for depression relative to non-asthmatics (Adjusted Odds Ratio $(A O R)=1.5$, $95 \%$ confidence interval $(\mathrm{CI})=1.2-1.8), \mathrm{p}=0.001)$. Those living in a single-family home had higher chance for depression than those living in 2-parents homes ( $\mathrm{AOR}=2.5,95 \% \mathrm{CI}=1.9-3.2), \mathrm{p}=0.001)$. Other predictors were lack of emotional support, age, poverty $<100 \%$, poor health, and smoking parent $(\mathrm{p}<0.05)$. DISCUSSION/ SIGNIFICANCE OF IMPACT: We concluded that depression was associated with asthma in children from poor single family who lack an emotional support. Management of asthma should include screening and management of psychosocial factors.

Bowel Ischemia after Continuous Flow Ventricular Assist Device Therapy: A Single Center Analysis

Ashley Y Choi ${ }^{1}$, Jatin Anand ${ }^{2}$, Muath Bishawi ${ }^{2}$,

Mani A. Daneshmand ${ }^{2}$, Jacob N. Schroder ${ }^{2}$, Suresh M. Agarwal ${ }^{2}$ and Carmelo A. Milano ${ }^{2}$

${ }^{1}$ Duke University and ${ }^{2}$ Duke University Hospital

OBJECTIVES/SPECIFIC AIMS: The purpose of the study was to describe patient characteristics associated with subsequent 